\title{
Collins effect and single spin azimuthal asymmetries in the HERMES and COMPASS experiments
}

\author{
A. V. Efremov, ${ }^{1}$ K. Goeke, ${ }^{2}$ and P. Schweitzer ${ }^{2}$ \\ 1 Joint Institute for Nuclear Research, Dubna, 141980 Russia \\ 2 Institut für Theoretische Physik II, Ruhr-Universität Bochum, D-44780 Bochum, Germany
}

(Dated: September 2003)

\begin{abstract}
Predictions are made for single spin azimuthal asymmetries due to the Collins effect in pion production from semi-inclusive deeply inelastic scattering off transversely and longitudinally polarized targets for the HERMES and COMPASS experiments. The $x$-dependence of the asymmetries is evaluated using the parton distribution functions from the chiral quark-soliton model. The overall normalization of the predicted asymmetries is determined by the information on the Collins fragmentation function extracted from previous HERMES data on azimuthal asymmetries $A_{U L}^{\sin \phi}$ from a longitudinally polarized target. The single spin asymmetries $A_{U T}$ from the transversely polarized proton target are found to be about $20 \%$ for positive and neutral pions both at HERMES and COMPASS. For a longitudinally polarized target we obtain for COMPASS $A_{U L}^{\sin \phi} \sim 1 \%$ and $A_{U L}^{\sin 2 \phi} \sim 3 \%$.

PACS numbers: 13.85.Ni, 13.60.Hb, 13.87.Fh, 13.88.+e
\end{abstract}

\section{INTRODUCTION}

Noticeable single spin azimuthal asymmetries ${ }^{1} A_{U L}^{\sin \phi}$ have been observed by the HERMES collaboration in pion and kaon electro-production in semi-inclusive deep-inelastic scattering (SIDIS) of an unpolarized lepton beam off a longitudinally polarized proton or deuteron target [1-4]. Recently the CLAS collaboration reported the measurement of the azimuthal asymmetry $A_{L U}^{\sin \phi}$ from SIDIS of a polarized beam off an unpolarized target [5]. Previously indications for the azimuthal asymmetry $A_{U T}$ from SIDIS of an unpolarized beam off a transversely polarized target were reported by the SMC collaboration [6].

Assuming factorization these single spin asymmetries can be explained by the Collins [7] and Sivers effect [8] in terms of so far unexplored distribution and fragmentation functions, namely the nucleon chirally odd twist-2 transversity distribution $h_{1}^{a}$ and twist-3 distribution functions $h_{L}^{a}$ and $e^{a}$ [9], the Collins fragmentation function $H_{1}^{\perp a}[7,10]$, the chirally even Sivers distribution function $f_{1 T}^{\perp a}[8,11-14]$ (and/or transverse momentum weighted moments thereof $[15,16])$. The $H_{1}^{\perp a}$ and $f_{1 T}^{\perp a}$ quantify the Collins and Sivers effect. The former describes the left-right asymmetry in the fragmentation of a transversely polarized quark into an unpolarized hadron; the latter describes the distribution of unpolarized quarks in a transversely polarized nucleon. Both are referred to as T-odd since, if there were no interactions, these functions would be forbidden by time reversal.

The HERMES data on single spin azimuthal asymmetries from SIDIS off a longitudinally polarized target [1-4] provide important indications that the mechanisms suggested by Collins and Sivers $[7,8]$ work, which makes them most exciting but also difficult to interpret. It is not clear which portion of the observed effect should be assigned to the Collins- and which to the Sivers mechanism. Moreover, numerous novel distribution- and fragmentation functions complicate the analysis. Reasonable descriptions of the HERMES data [1-4] using different assumptions and models were given in Refs. [17-23] in terms of the Collins effect only. Noteworthy, information on the Sivers function gained from phenomenological description of single spin asymmetries in $p p^{\uparrow} \rightarrow \pi X$ [14] indicates that neglecting the Sivers effect in the analysis of the HERMES experiment could be justified [24].

The understanding of the underlying phenomena is difficult also because so far there is only one clear observable for target single spin asymmetries in SIDIS with polarized targets, the $A_{U L}^{\sin \phi}$ asymmetry measured by HERMES [1-4]. Although at HERMES $A_{U L}^{\sin \phi}$ was measured in electro-production of different hadrons from different targets - providing valuable insights into the flavour dependence of the process - the observation of other independent observables which allow to distinguish the Collins and Sivers effect is needed to clarify the situation.

The azimuthal asymmetry $A_{U L}^{\sin 2 \phi}$ is such an observable, for it is generated by the Collins effect only [15, 16]. Unfortunately, in the kinematics of the HERMES experiment $A_{U L}^{\sin 2 \phi}$ was found rather small and consistent with zero

\footnotetext{
${ }^{1} U$ denotes the unpolarized beam. $L$ (below also $T$ ) denotes the longitudinal (and transverse) target polarization with respect to the beam. The superscript $\sin \phi$ characterizes the azimuthal distribution of the produced hadrons with respect to the direction of the exchanged virtual photon. The precise definitions are given in the Appendix.
} 


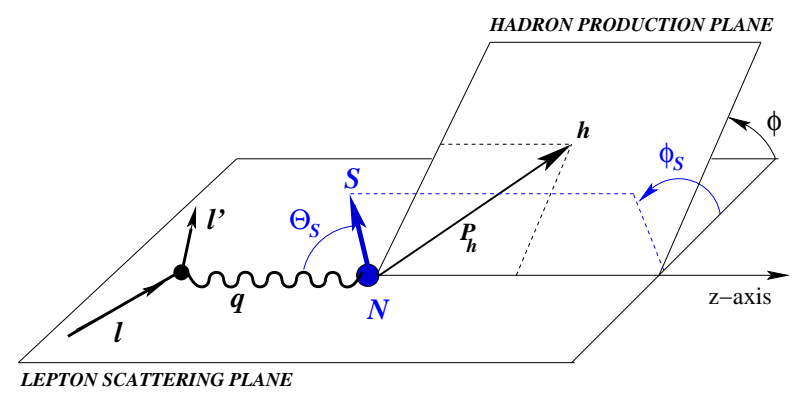

FIG. 1: Kinematics of the process $l N^{\uparrow} \rightarrow l^{\prime} h X$ in the lab frame.

within (relatively large) error bars [1-4]. This asymmetry could be accessed in the CLAS experiment, which operates at somehow lower energies and higher luminosity than HERMES. In the different kinematics of the CLAS experiment $A_{U L}^{\sin 2 \phi}$ is expected to be larger than at HERMES and measurable $[25],{ }^{2}$ and, indeed, encouraging preliminary results have already been reported in Ref. [26]. Also in the COMPASS experiment $A_{U L}^{\sin 2 \phi}$ will probably be observable - as we will estimate below.

More conclusive insights, however, are expected from SIDIS experiments with transversely polarized targets ${ }^{3}$, where the Collins and Sivers effects [7, 8], can cleanly be distinguished [16]. Those experiments are presently in progress at HERMES [27] and COMPASS [28]. Estimates of these asymmetries for HERMES were presented in Refs. [21, 29].

In this paper we will predict the azimuthal single spin asymmetry due to the Collins effect from a transversely polarized target for the kinematics of the HERMES and COMPASS experiments. For that we shall use predictions for the transversity distribution function $h_{1}^{a}(x)$ from the chiral quark-soliton model [30] and information on the analyzing power $\left\langle H_{1}^{\perp}\right\rangle /\left\langle D_{1}\right\rangle$ from a previous analysis [22] of the HERMES data. ${ }^{4}$ Indeed, the present approach, based on the chiral-quark soliton model and the instanton vacuum picture, describes in a theoretically consistent and phenomenologically satisfying way $[22,23]$ the $x$-dependence of the HERMES data [1-4]. In a certain sense the analyzing power $\left\langle H_{1}^{\perp}\right\rangle /\left\langle D_{1}\right\rangle$ from [22] quantifies the amount of Collins effect needed to understand the HERMES data [1-4] within this approach. Therefore the comparison of our prediction to the outcome of the HERMES and COMPASS transverse target polarization experiments will yield more than an important test of the approach and its consistency. An agreement would support also the conclusion of Ref. [24] that the Sivers effect can be neglected in $A_{U L}^{\sin \phi}$-asymmetries and it would justify, a posteriori, the attempts [17-23] to understand HERMES data on $A_{U L}$ in terms of the Collins effect only.

The paper is organized as follows. In Section II the SIDIS process and its description is discussed under the assumption of factorization. In Section III our assumptions on the novel distribution and fragmentation functions are described. In Sections IV and V the predictions are presented for the HERMES and COMPASS transverse target polarization experiments, as well as for the longitudinal target polarization experiment at COMPASS. In Section VI we present general comments on the Sivers effect in SIDIS asymmetries. Section VII contains the summary and conclusions.

\section{THE CONTRIBUTION OF THE COLLINS EFFECT TO THE AZIMUTHAL ASYMMETRY FROM A TRANSVERSELY POLARIZED TARGET}

In the HERMES and COMPASS experiments the cross sections $\sigma_{N}^{\uparrow \downarrow}$ for the process $l N^{\uparrow \downarrow} \rightarrow l^{\prime} h X$ will be measured, where $N^{\uparrow \downarrow}$ denotes the transversely with respect to the beam polarized target, see Fig. 1.

With $P, l$ and $l^{\prime}$ denoting the momenta of the target, incoming and outgoing lepton the kinematic variables are

\footnotetext{
2 The different kinematics and high luminosity at CLAS have already been explored to measure another asymmetry previously found consistent with zero at HERMES, namely the azimuthal asymmetry in SIDIS of a polarized beam off an unpolarized target, $A_{L U}^{\text {sin } \phi}$. This asymmetry could be due to the Collins effect $[15,16]$ and provide first indications to the twist-3 distribution function $e^{a}(x)[25]$.

${ }^{3}$ A first observation of single spin azimuthal asymmetries in SIDIS from a transversely polarized target - which unfortunately retained its preliminary status - was reported from the SMC experiment [6].

4 Actually, in that analysis [22] the Sivers function was neglected, which has later been shown to be theoretically consistent and phenomenologically justified [24].
} 
defined as $s:=(P+l)^{2}, q:=l-l^{\prime}$ with $Q^{2}:=-q^{2}$, and $W^{2}:=(P+q)^{2}$, and

$$
x:=\frac{Q^{2}}{2 P q}, \quad y:=\frac{P q}{P l}, \quad z:=\frac{P P_{h}}{P q} .
$$

Let $S^{\uparrow \downarrow}$ denote the modulus of the polarization vector. The component of the target polarization vector which is transverse with respect to the hard photon is characterized by the angle $\Theta_{S}$, see Fig. 1, given by

$$
\sin \Theta_{S}=\frac{S_{T}^{\uparrow \downarrow}}{S^{\uparrow \downarrow}}=\cos \theta_{\gamma} \sqrt{1+\tan ^{2} \theta_{\gamma} \sin ^{2} \phi_{S^{\prime}}} \approx \cos \theta_{\gamma},
$$

where $\phi_{S}^{\prime}$ is the azimuthal angle of the target spin direction around the lepton beam direction counted from the scattering plane, and $\cos \theta_{\gamma}$ is given by

$$
\cos \theta_{\gamma}=\sqrt{1-\frac{\left(4 M_{N}^{2} x^{2}\right)\left(1-y-M_{N}^{2} x^{2} y^{2} / Q^{2}\right)}{\left(Q^{2}+4 M_{N}^{2} x^{2}\right)}} .
$$

Since $\tan ^{2} \theta_{\gamma}=\mathcal{O}\left(M^{2} / Q^{2}\right)$ the approximation in the last step of Eq. (3) works well.

With $\phi\left(\phi_{S}\right)$ denoting the azimuthal angles between the hadron production plane (the nucleon spin) and the lepton scattering plane, see Fig. 1, the observables of interest are defined as

$$
\begin{aligned}
A_{U T}^{\sin \left(\phi+\phi_{S}\right)}(x) & =\frac{\int \mathrm{d} z \mathrm{~d} y \mathrm{~d}^{2} \mathbf{P}_{h \perp} \sin \left(\phi+\phi_{s}\right)\left(\frac{1}{S^{\uparrow}} \frac{\mathrm{d}^{5} \sigma^{\uparrow}}{\mathrm{d} x \mathrm{~d} y \mathrm{~d} z \mathrm{~d}^{2} \mathbf{P}_{h \perp}}-\frac{1}{S^{\downarrow}} \frac{\mathrm{d}^{5} \sigma^{\downarrow}}{\mathrm{d} x \mathrm{~d} y \mathrm{~d} z \mathrm{~d}^{2} \mathbf{P}_{h \perp}}\right)}{\frac{1}{2} \int \mathrm{d} z \mathrm{~d} y \mathrm{~d}^{2} \mathbf{P}_{h \perp}\left(\frac{\mathrm{d}^{5} \sigma^{\uparrow}}{\mathrm{d} x \mathrm{~d} y \mathrm{~d} z \mathrm{~d}^{2} \mathbf{P}_{h \perp}}+\frac{\mathrm{d}^{5} \sigma^{\downarrow}}{\mathrm{d} x \mathrm{~d} y \mathrm{~d} z \mathrm{~d}^{2} \mathbf{P}_{h \perp}}\right)} \\
A_{U T}^{\sin \left(\phi+\phi_{S}\right) k_{\perp} /\left\langle P_{h \perp}\right\rangle}(x) & =\frac{\int \mathrm{d} z \mathrm{~d} y \mathrm{~d}^{2} \mathbf{P}_{h \perp} \sin \left(\phi+\phi_{s}\right) \frac{k_{\perp}}{\left\langle P_{h \perp}\right\rangle}\left(\frac{1}{S^{\uparrow}} \frac{\mathrm{d}^{5} \sigma^{\uparrow}}{\mathrm{d} x \mathrm{~d} y \mathrm{~d} z \mathrm{~d}^{2} \mathbf{P}_{h \perp}}-\frac{1}{S^{\downarrow}} \frac{\mathrm{d}^{5} \sigma^{\downarrow}}{\mathrm{d} x \mathrm{~d} y \mathrm{~d} z \mathrm{~d}^{2} \mathbf{P}_{h \perp}}\right)}{\frac{1}{2} \int \mathrm{d} z \mathrm{~d} y \mathrm{~d}^{2} \mathbf{P}_{h \perp}\left(\frac{\mathrm{d}^{5} \sigma^{\uparrow}}{\mathrm{d} x \mathrm{~d} y \mathrm{~d} z \mathrm{~d}^{2} \mathbf{P}_{h \perp}}+\frac{\mathrm{d}^{5}}{\mathrm{~d} x \mathrm{~d} y \mathrm{~d} z \mathrm{~d}^{2} \mathbf{P}_{h \perp}}\right)} .
\end{aligned}
$$

The weight $\sin \left(\phi+\phi_{s}\right)$ in Eq. (4) has the drawback to leave convoluted the transverse momenta in the unintegrated distribution and fragmentation functions - in this case $h_{1}\left(x, P_{\mathrm{N} \perp}^{2}\right)$ and $H_{1}^{\perp}\left(z, k_{T}^{2}\right)[15]$. (For the meaning and definition of unintegrated distribution functions in QCD see [31] and references therein.) The additional power of transverse momentum ${ }^{5} k_{\perp}=\left|\mathbf{P}_{h \perp}\right| / z$ in the weight in Eq. (5) yields expressions where the transverse momenta are disentangled in a model independent way [16].

Though the asymmetry weighted with $k_{\perp}$ in Eq. (5) is preferable from a theoretical point of view [16], we shall consider both asymmetries, Eq. (4) and Eq. (5). Considering also the asymmetry (4) will allow us to directly compare the predicted effect to the $A_{U L}^{\sin \phi}$ asymmetries measured at HERMES [1-4] which were analyzed in a way analogous to Eq. (4).

The expressions for the differential cross sections entering the asymmetries in Eqs. (4, 5) were derived in [15] assuming factorization. In order to deconvolve the transverse momenta in $A_{U T}^{\sin \left(\phi+\phi_{S}\right)}$ in Eq. (4) we assume the distributions of transverse momenta in the unintegrated distribution and fragmentation functions to be Gaussian. This ansatz is in fair agreement with the HERMES data in the case of $A_{U L}^{\sin \phi}$ asymmetries [1-4]. Under this assumption one obtains [15] (cf. also [22])

$$
A_{U T}^{\sin \left(\phi+\phi_{s}\right)}(x)=a_{\text {Gauss }} B_{T}(x) \frac{\sum_{a} e_{a}^{2} x h_{1}^{a}(x)\left\langle H_{1}^{\perp a}\right\rangle}{\sum_{b} e_{b}^{2} x f_{1}^{b}(x)\left\langle D_{1}^{b}\right\rangle},
$$

while the result for the $k_{\perp}$-weighted asymmetry is given by [16]

$$
A_{U T}^{\sin \left(\phi+\phi_{s}\right) k_{\perp} /\left\langle P_{h \perp}\right\rangle}(x)=B_{T}(x) \frac{\sum_{a} e_{a}^{2} x h_{1}^{a}(x)\left\langle H_{1}^{\perp(1) a}\right\rangle}{\sum_{b} e_{b}^{2} x f_{1}^{b}(x)\left\langle D_{1}^{b}\right\rangle},
$$

5 We use the notation of $[15,16]$ with $H_{1}^{\perp}$ normalized to $\left\langle P_{h \perp}\right\rangle$ instead of $m_{h}$. Correspondingly we choose $\left\langle P_{h \perp}\right\rangle$ to compensate the dimension of $k_{\perp}$ in Eq. (5). 
where $B_{T}(x)$ and $a_{\text {Gauss }}$ are defined as (experimental cuts have to be considered in the integration over $y$ )

$$
\begin{aligned}
B_{T}(x) & =\frac{2 \int \mathrm{d} y(1-y) \sin \Theta_{S} / Q^{4}}{\int \mathrm{d} y\left(1-y+y^{2} / 2\right) / Q^{4}}, \\
a_{\text {Gauss }} & =\frac{1}{2\langle z\rangle \sqrt{1+\left\langle z^{2}\right\rangle\left\langle P_{\mathrm{N} \perp}^{2}\right\rangle /\left\langle P_{h \perp}^{2}\right\rangle}},
\end{aligned}
$$

where $\left\langle P_{\mathrm{N} \perp}^{2}\right\rangle$ and $\left\langle P_{h \perp}^{2}\right\rangle /\left\langle z^{2}\right\rangle$ are the mean transverse momentum squares characterizing the Gaussian distributions of transverse momenta in the unintegrated distribution and fragmentation function. The prefactor $a_{\text {Gauss }}$ contains the model dependence; it would be different if we assumed the distributions of transverse momenta to be different from Gaussian. $H_{1}^{\perp(1) a}(z)$ in Eq. (7) is defined by [16] (cf. footnote 5)

$$
H_{1}^{\perp(1) a}(z)=\int \mathrm{d}^{2} \mathbf{k}_{T} \frac{z^{2} \mathbf{k}_{T}^{2}}{2\left\langle P_{h \perp}\right\rangle^{2}} H_{1}^{\perp a}\left(z, z^{2} \mathbf{k}_{T}^{2}\right) .
$$

\section{TRANSVERSITY DISTRIBUTION AND COLLINS FRAGMENTATION FUNCTION}

In order to estimate the azimuthal asymmetries, Eqs. $(4,5)$ and $(6,7)$, one has to know $h_{1}^{a}$ and $H_{1}^{\perp a}$. For the former we shall use the predictions of the chiral quark-soliton model ( $\chi \mathrm{QSM})$ [30], and for the latter our analysis of the HERMES data from Ref. [22].

Chirally and T-odd distribution functions. The $\chi \mathrm{QSM}$ is an effective relativistic quantum field-theoretical model with explicit quark degrees of freedom, in which twist-2 nucleon distribution functions can unambiguously be defined and evaluated at a low renormalization point of about $(600-700) \mathrm{MeV}$. The $\chi$ QSM has been derived from the instanton model of the QCD vacuum [32] and has been shown to describe well numerous static nucleonic observables without adjustable parameters [33]. The field-theoretical nature of the model is crucial to ensure the theoretical consistency of the approach: the quark and antiquark distribution functions computed in the model satisfy all general QCD requirements [34]. The results of the model agree for the distribution functions $f_{1}^{a}(x), g_{1}^{a}(x)$ and $g_{T}^{a}(x)[34-$ 36 ] within $(10-30) \%$ with phenomenological information. This encourages confidence that the model describes the nucleon transversity distribution function $h_{1}^{a}(x)[30]$ with a similar accuracy.

In the following we will need also the deuteron transversity distribution. Corrections due to the D-state admixture were estimated to be very similar to the corresponding corrections in the case of the helicity distribution function [37]. Since these corrections are smaller than other theoretical uncertainties in our approach we shall disregard them here and estimate e.g. for the $u$-quark

$$
h_{1}^{u / D}(x) \approx h_{1}^{u / p}(x)+h_{1}^{u / n}(x)=h_{1}^{u}(x)+h_{1}^{d}(x)
$$

where isospin symmetry was used in the last step, and $h_{1}^{u}(x)$ and $h_{1}^{d}(x)$ refer (as always) to the proton.

In the $\chi \mathrm{QSM} h_{1}^{a}(x) \neq g_{1}^{a}(x)$ already at a low normalization point. However, due to the large error bars the present data do not discriminate between different models. Therefore reasonable descriptions of the $A_{U L}^{\sin \phi}$ asymmetries have also been obtained assuming $h_{1}^{a}(x)=g_{1}^{a}(x)$ being motivated by the non-relativistic quark model or using other models [20]. An advantage of relying on predictions from the $\chi$ QSM (based on the instanton vacuum) lies in the fact that all novel distribution functions are taken from an approach, which is internally consistent and which has been shown in many different observables to be reasonable. For example, in the instanton vacuum model the pure twist-3 contribution $\widetilde{h}_{L}^{a}(x)$ to $h_{L}^{a}(x)$ is strongly suppressed [38]. Thus in this approach one can justifiably approximate $h_{L}^{a}(x)$ by its twist-2 ("Wandzura-Wilczek" like) term $h_{L}^{a}(x)=2 x \int_{x}^{1} \mathrm{~d} x^{\prime} h_{1}^{a}\left(x^{\prime}\right) / x^{\prime 2}$. Moreover, T-odd distribution functions vanish in the $\chi$ QSM (as they do in a large class of other chiral soliton models) [39]. Therefore in this approach it is consistent to neglect the Sivers effect in $A_{U L}^{\sin \phi}$ asymmetries [24], see also Section VI for further comments.

In Refs. $[22,23]$ the present approach has been shown to describe well the $x$-dependence of $A_{U L}$ from the HERMES longitudinal target polarization experiments [1-4].

The Collins fragmentation function. Let us define the favoured Collins fragmentation function as

$$
H_{1}^{\perp} \equiv H_{1}^{\perp u / \pi^{+}}=H_{1}^{\perp \bar{d} / \pi^{+}}=H_{1}^{\perp d / \pi^{-}}=2 H_{1}^{\perp u / \pi^{0}}=\ldots \text { etc. } \gg H_{1}^{\perp d / \pi^{+}}=H_{1}^{\perp \bar{u} / \pi^{+}}=\ldots \text { etc. }
$$

The equalities in Eq. (12) follow from charge conjugation and isospin symmetry. The strong suppression of the unfavoured with respect to the favoured Collins fragmentation function has been concluded on the basis of the Schäfer-Teryaev sum rule [40]. 
In Ref. [22] information on $H_{1}^{\perp}$ was gained from the HERMES data on the $A_{U L}^{\sin \phi}$ asymmetry in $\pi^{+}$and $\pi^{0}$ production $[2,3]$. For that the transverse momentum distributions were assumed to be Gaussian and the parton distribution functions $h_{1}^{a}$ and $h_{L}^{a}$ were taken from the chiral quark soliton model. For the analyzing power the value was found ( $D_{1}$ denotes the favoured unpolarized fragmentation function)

$$
\left\langle H_{1}^{\perp}\right\rangle /\left\langle D_{1}\right\rangle=(13.8 \pm 2.8) \%
$$

at $\langle z\rangle=0.4$ and $\left\langle Q^{2}\right\rangle=2.5 \mathrm{GeV}^{2}[22]$. The result in Eq. (13) contains - apart from the shown statistical error from the HERMES experiment - further uncertainties due to the systematic error of the HERMES experiment and model dependence. These errors need not be considered in the following, when the above result is used to make predictions for $A_{U T}^{\sin \left(\phi+\phi_{S}\right)}$ in the HERMES experiment in combination with results from the chiral quark-soliton model and the instanton vacuum model. In a certain sense the result in Eq. (13) can be considered as a fit to the $A_{U L}^{\text {sin } \phi}$ data [2, 3]. Noteworthy, a result numerically close to Eq. (13) was obtained in the model calculation of Ref. [29].

In $e^{+} e^{-}$annihilation the Collins effect can give rise to a specific azimuthal asymmetry of a hadron in a jet around the axis in the direction of the second hadron in the opposite jet. This asymmetry was measured using the DELPHI data collection and a value $\left|\left\langle H_{1}^{\perp}\right\rangle /\left\langle D_{1}\right\rangle\right|=(12.5 \pm 1.4) \%$ for $\langle z\rangle \simeq 0.4$ at a scale of $M_{Z}^{2}$ was reported [41, 42]. ${ }^{6}$ In previous works $[22,23]$ this value (assuming a positive sign) was used to analyze the HERMES data [1-4]. For that the scale dependence of the ratio $\left\langle H_{1}^{\perp}\right\rangle /\left\langle D_{1}\right\rangle$ was assumed to be weak and possible Sudakov suppression effects [43] were neglected. However, as shown in Ref. [44] the Collins fragmentation function could be process-dependent, i.e. different in $e^{+} e^{-}$annihilation and SIDIS.

Therefore, in this note we shall use the result in Eq. (13) extracted from SIDIS HERMES data. Numerically the difference is not relevant - from a theoretical point of view, however, the use of the result in Eq. (13) is preferable for our purpose to describe SIDIS processes.

\section{COLLINS $A_{U T}$ ASYMMETRIES IN THE HERMES EXPERIMENT}

The asymmetry $A_{U T}^{\sin \left(\phi+\phi_{S}\right)}$. In order to estimate $A_{U T}^{\sin \left(\phi+\phi_{S}\right)}$ in the HERMES experiment we rely on the same assumptions and approximations which were used in Refs. [22, 23] to analyze the HERMES data on the $A_{U L}^{\text {sin } \phi}$ asymmetries. In particular we assume a Gaussian distribution of transverse momenta (cf. Section II), take $h_{1}^{a}(x)$ from the $\chi$ QSM and $H_{1}^{\perp}$ from our previous analysis of HERMES-data [24] and assume favoured fragmentation - as described in Section III. For the unpolarized distribution functions $f_{1}^{a}(x)$ we use the parameterization of Ref [45]. For the parameter characterizing the (Gaussian) distribution of transverse momenta in the nucleon we shall use the estimate $\left\langle P_{\mathrm{N} \perp}\right\rangle=0.4 \mathrm{GeV}$ from Refs. [46, 47]. The result, however, is only weakly sensitive to the actual choice for this parameter.

The beam in the HERMES experiment has an energy of $E_{\text {beam }}=26.7 \mathrm{GeV}$. We assume the cuts implicit in the integrations in Eq. (8) to be the same as in the longitudinal target polarization experiments [1-4]

$$
1 \mathrm{GeV}^{2}<Q^{2}<15 \mathrm{GeV}^{2}, \quad 2 \mathrm{GeV}<W, \quad 0.2<y<0.85, \quad 0.023<x<0.4,
$$

and $0.2<z<0.7$ with $\langle z\rangle=0.4$, and $\left\langle P_{h \perp}\right\rangle=0.4 \mathrm{GeV}$. Note that strictly speaking we neglect the implicit dependence of distribution and fragmentation functions on $y$ through the scale $Q^{2}=x y\left(s-M_{N}^{2}\right)$, and evaluate them instead at the average scale in the HERMES experiment $\left\langle Q^{2}\right\rangle=2.5 \mathrm{GeV}^{2}$. The predictions for $A_{U T}^{\sin \left(\phi+\phi_{S}\right)}$ for the transversely polarized proton and deuterium target are shown in Figs. 2a and 2b, respectively.

Figs. $2 \mathrm{a}$ and $2 \mathrm{~b}$ demonstrate that $A_{U T}^{\sin \left(\phi+\phi_{S}\right)}$ is sizeable, roughly $20 \%$ for positive and neutral pions for the proton target and about $10 \%$ for all pions for the deuteron target. Comparing this result with the $A_{U L}^{\text {sin } \phi}$ asymmetries $\sim(2-4) \%[1-4]$ we see that $A_{U T}^{\sin \left(\phi+\phi_{S}\right)}$ asymmetry can clearly be observed, cf. [21]. A comparably large value for this asymmetry was estimated in [29] on the basis of a model calculation for the Collins function and assuming $h_{1}^{a}(x)$ to saturate the Soffer bound [48].

The accuracy of the predictions - for $\pi^{+}$and $\pi^{0}$ asymmetries from a proton target - is mainly determined by the theoretical uncertainty of the $\chi$ QSM prediction for $h_{1}^{a}(x)$ of about $20 \%$ and the statistical error of the analyzing power (13) from the HERMES experiment. For negative pions from a proton, however, there might be additional sizeable

\footnotetext{
6 This result is referred to as "more optimistic" since it is subject to presumably larger systematic uncertainties than the "more reliable" value $\left|\left\langle H_{1}^{\perp}\right\rangle /\left\langle D_{1}\right\rangle\right|=(6.3 \pm 2.0) \%$ reported in $[41,42]$ which has presumably smaller systematic errors. For both values no estimate of systematic errors could be given in $[41,42]$.
} 
$\mathbf{A}_{\mathbf{U T}}^{\sin \left(\phi+\phi_{s}\right)}(\mathbf{x})$ at HERMES

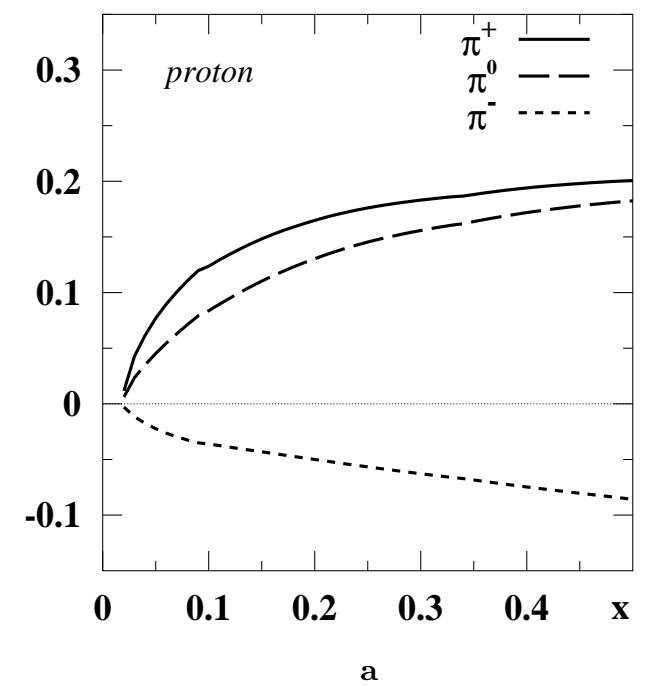

$\mathbf{A}_{\mathbf{U T}}^{\sin \left(\phi+\phi_{\mathrm{s}}\right)}(\mathbf{x})$ at HERMES

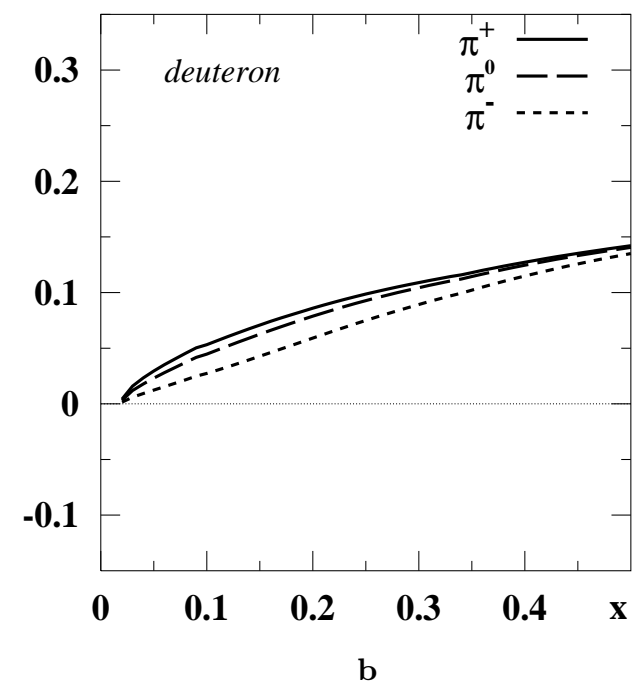

FIG. 2: Predictions for azimuthal asymmetries $A_{U T}^{\sin \left(\phi+\phi_{S}\right)}(x)$ in SIDIS pion productions from transversely polarized proton (a) and deuteron (b) targets for kinematics of the HERMES experiment.

corrections due to unfavoured flavour fragmentation [20]. In this case the small unfavoured Collins fragmentation function is multiplied by the large $\frac{4}{9} h_{1}^{u}(x)$ while the large favoured fragmentation function is multiplied by the small $\frac{1}{9} h_{1}^{d}(x) .{ }^{7}$ Therefore $\pi^{-}$is more sensitive to corrections due to unfavoured fragmentation than $\pi^{+}$and $\pi^{0}$ where $u$ quark dominance $\left(h_{1}^{u}(x) \gg\left|h_{1}^{d}(x)\right|\right)$ tends to enhance the favoured fragmentation effect. Similar reservations apply to the deuteron target where there is no $u$-quark dominance - apart from that introduced by the quark electric charges.

In Ref. [23] $A_{U L}^{\sin \phi}$ asymmetries for kaons have been estimated assuming that the analyzing power for kaons is approximately equal to that of pions, i.e. $\left\langle H_{1}^{\perp}\right\rangle /\left.\left\langle D_{1}\right\rangle\right|_{\text {kaon }} \approx\left\langle H_{1}^{\perp}\right\rangle /\left.\left\langle D_{1}\right\rangle\right|_{\text {pion. }}$. This relation would hold exactly in the chiral limit (where pions and kaons would be massless Goldstone bosons). The kaon $A_{U L}^{\sin \phi}$ asymmetries predicted in [23] on the basis of this assumption compare well with the HERMES data within the (admittedly rather large) statistical error [4]. Under this assumption one could expect for the transverse target polarization experiment (cf. Ref. [23] for further details)

$$
\begin{aligned}
& A_{U T}^{\sin \left(\phi+\phi_{S}\right)}\left(K^{+}\right) \approx A_{U T}^{\sin \left(\phi+\phi_{S}\right)}\left(K^{0}\right) \approx A_{U T}^{\sin \left(\phi+\phi_{S}\right)}\left(\pi^{+}\right), \\
& A_{U T}^{\sin \left(\phi+\phi_{S}\right)}\left(\bar{K}^{0}\right) \approx A_{U T}^{\sin \left(\phi+\phi_{S}\right)}\left(K^{-}\right) \approx 0 .
\end{aligned}
$$

The asymmetry $A_{U T}^{\sin \left(\phi+\phi_{S}\right) k_{\perp} /\left\langle P_{h \perp}\right\rangle}$. In this case we need the transverse momentum weighted moment of the favoured Collins fragmentation function $H_{1}^{\perp(1)}$, see Eq. (7). Under the assumption of a Gaussian distribution of transverse momenta in Eq. (10) one obtains for $H_{1}^{\perp(1)}$ averaged over $z$

$$
\left\langle H_{1}^{\perp(1)}\right\rangle=\frac{\left\langle P_{h \perp}^{2}\right\rangle}{2\left\langle P_{h \perp}\right\rangle^{2}}\left\langle H_{1}^{\perp}\right\rangle=\frac{2}{\pi}\left\langle H_{1}^{\perp}\right\rangle,
$$

where we used the relation $\left\langle P_{h \perp}^{2}\right\rangle /\left\langle P_{h \perp}\right\rangle^{2}=4 / \pi$ valid for a Gaussian distribution. The $\left\langle H_{1}^{\perp}\right\rangle$ in Eq. (16) can be taken from Eq. (13) (recall that the analyzing power (13) was extracted under the assumption of a Gaussian transverse momentum distribution [23]).

Therefore we obtain the relation

$$
A_{U T}^{\sin \left(\phi+\phi_{S}\right) k_{\perp} /\left\langle P_{h \perp}\right\rangle}=\beta_{\text {Gauss }} A_{U T}^{\sin \left(\phi+\phi_{S}\right)} .
$$

\footnotetext{
7 The antiquark distributions can be disregarded for this qualitative consideration. The same applies to unpolarized fragmentation. Since $f_{1}^{a}(x)$ and $D_{1}^{a}(z)$ are positive, the effect of unpolarized unfavoured fragmentation may decrease the asymmetry but cannot change its sign - as could do the polarized unfavoured fragmentation in the case of $\pi^{-}$from a proton target [20].
} 
The constant $\beta_{\text {Gauss }}$ "converts" between the differently weighted asymmetries and is given by

$$
\beta_{\text {Gauss }}=\frac{2}{\pi a_{\text {Gauss }}}=\frac{4\langle z\rangle}{\pi} \sqrt{1+\left\langle z^{2}\right\rangle\left\langle P_{\mathrm{N} \perp}^{2}\right\rangle /\left\langle P_{h \perp}^{2}\right\rangle} \approx 0.55
$$

for the numbers in the HERMES experiment. Thus, in order to obtain our prediction for $A_{U T}^{\sin \left(\phi+\phi_{S}\right) k_{\perp} /\left\langle P_{h \perp}\right\rangle}$ it is sufficient to multiply the results in Figs. $2 \mathrm{a}$ and $2 \mathrm{~b}$ by the factor 0.55 . We stress that the "conversion factor" $\beta_{\mathrm{Gauss}}$ is model dependent. For a different model of transverse momenta $\beta_{\text {model }} \neq \beta_{\text {Gauss }}$. In particular, $\beta_{\text {model }}$ could numerically be different from the result in Eq. (18).

Our prediction for $A_{U T}^{\sin \left(\phi+\phi_{S}\right)}$ is more robust than that for $A_{U T}^{\sin \left(\phi+\phi_{S}\right) k_{\perp} /\left\langle P_{h \perp}\right\rangle}(x)$ since the latter - in addition to other assumptions in our approach - also tests the assumption of a Gaussian transverse momentum distribution. In fact, the only assumption entering our prediction for $A_{U T}^{\sin \left(\phi+\phi_{S}\right)}$ - and the analysis of $A_{U L}^{\sin \phi}$ in Ref. [23] - is that a generic unintegrated fragmentation function $F\left(z, \mathbf{k}_{T}^{2}\right)$ can be approximated by

$$
F\left(z, \mathbf{k}_{T}^{2}\right) \approx F(z) G\left(\mathbf{k}_{T}^{2}\right)
$$

where $G\left(\mathbf{k}_{T}^{2}\right)$ satisfies $\int \mathrm{d}^{2} \mathbf{k}_{T} G\left(\mathbf{k}_{T}^{2}\right)=1$, and analogous for unintegrated distribution functions. For a Gaussian distribution one sets $G\left(\mathbf{k}_{T}^{2}\right)=\exp \left(-\mathbf{k}_{T}^{2} /\left\langle\mathbf{k}_{T}^{2}\right\rangle\right) /\left(\pi\left\langle\mathbf{k}_{T}^{2}\right\rangle\right)$. Assuming (19) but taking a different model for $G\left(\mathbf{k}_{T}^{2}\right)$ we would obtain a different constant $a_{\text {model }} \neq a_{\text {Gauss }}$ in Eq. (9). With a different model for transverse momenta, however, we also would have obtained a different result in Eq. (13) for $\left\langle H_{1}^{\perp}\right\rangle_{\text {model }}$. (In this context the $\left\langle H_{1}^{\perp}\right\rangle$ in Eq. (13) should be labelled $\left\langle H_{1}^{\perp}\right\rangle_{\text {Gauss }}$ for clarity.) Thus under the assumption (19) the relation $A_{U T}^{\sin \left(\phi+\phi_{S}\right)} \propto a_{\text {model }}\left\langle H_{1}^{\perp}\right\rangle_{\text {model }}$ is model independent. Therefore our predictions for $A_{U T}^{\sin \left(\phi+\phi_{S}\right)}$ shown in Figs. 2a and $2 \mathrm{~b}$ do not depend on the Gaussian model but rely solely on the the assumption (19). If the assumption (19) held one could discriminate between different models for the transverse momentum distributions by considering different powers of transverse momentum in the weight $\sin \left(\phi+\phi_{S}\right)\left|\mathbf{k}_{\perp}\right|^{n}(n=0,1)$. Considering different weights could provide interesting phenomenological insights. However, from a strict theoretical point of view the weighting with an adequate power of $\left|\mathbf{k}_{\perp}\right|$ is preferable [16].

Preliminary SMC results. Though devoted to the HERMES experiment let us conclude this section with a comment on the preliminary SMC data reported in Ref. [6]. In the SMC experiment indications were found that the transverse target spin asymmetry $\propto \sin \phi_{c} A_{N}$ with $A_{N}=0.11 \pm 0.06$, where the Collins angle $\phi_{c} \equiv \phi+\phi_{S}-\pi$ (cf. [6] for the precise definition of $A_{N}$ ). Our approach yields $A_{N}=-0.12$, i.e. an asymmetry of opposite sign [22, 23] (due to $\left.\sin \phi_{c}=-\sin \left(\phi+\phi_{S}\right)\right)$. Considering the preliminary status of the data of Ref. [6] it is not possible to draw any conclusions at this stage.

\section{COMPASS EXPERIMENT}

Transverse target spin asymmetry. The beam energy available at COMPASS is $E_{\text {beam }}=160 \mathrm{GeV}[28]$. For the kinematic cuts we shall take

$$
2 \mathrm{GeV}^{2}<Q^{2}<50 \mathrm{GeV}^{2}, \quad 15 \mathrm{GeV}^{2}<W^{2}<300 \mathrm{GeV}^{2}, \quad 0.05<y<0.9, \quad x<0.4,
$$

and evaluate the distribution functions at $Q^{2}=10 \mathrm{GeV}^{2}$. We take $\left\langle P_{h \perp}\right\rangle \approx 0.4 \mathrm{GeV}$ and $\langle z\rangle \approx 0.4$. The latter means that we can use for $\left\langle H_{1}^{\perp}\right\rangle /\left\langle D_{1}\right\rangle$ the result in Eq. (13) - if we assume that the ratio $\left\langle H_{1}^{\perp}\right\rangle /\left\langle D_{1}\right\rangle$ is only weakly scale dependent in the range of scales relevant in the HERMES and COMPASS experiments. The estimate of $A_{U T}^{\sin \left(\phi+\phi_{S}\right)}$ obtained in this way is shown in Fig. 3a.

In the HERMES experiment the analyzing power (assuming our approach) is $H_{1}^{\perp}(z) / D_{1}(z) \approx a z$ where the constant $a \approx \frac{1}{3}[22]$. This means that $\left\langle H_{1}^{\perp}\right\rangle /\left\langle D_{1}\right\rangle \approx a\langle z\rangle$. If such a pattern held also at COMPASS energies, it would be preferable to choose a larger low- $z$ cut in order to increase $\langle z\rangle$ and thus the analyzing power $\left\langle H_{1}^{\perp}\right\rangle /\left\langle D_{1}\right\rangle($ at the price of a lower statistics) [28]. For a different $\langle z\rangle$ the results shown in Fig. 3a have to be rescaled appropriately.

Fig. 3a shows that $A_{U T}^{\sin \left(\phi+\phi_{S}\right)}$ can be of $\mathcal{O}(20 \%)$ at COMPASS energies, i.e. as large as at HERMES. This is not unexpected since this asymmetry is twist-2 (in the sense that it is not power suppressed). Thus, the COMPASS experiment can equally well shed some light on the dynamics of the Collins effect. Actually, the theoretical accuracy of the predictions in Fig. 3a is less than in the case of the predictions for HERMES presented in the previous Section because one has to consider the uncertainty introduced by assuming that the HERMES value for the analyzing power (13) can be used at COMPASS energies. 

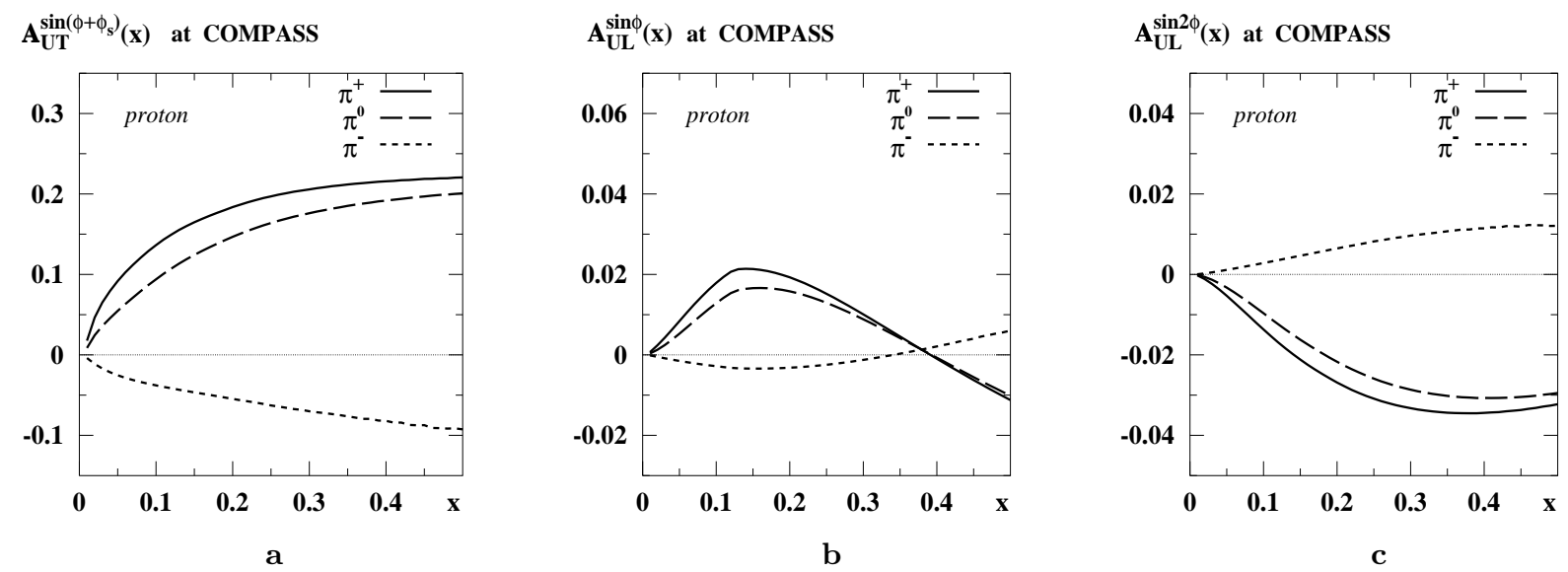

FIG. 3: a. Prediction of the azimuthal asymmetry $A_{U T}^{\sin \left(\phi+\phi_{S}\right)}(x)$ in SIDIS pion production from a transversely polarized proton target for the kinematics of the COMPASS experiment. Predictions of the azimuthal asymmetries $A_{U L}^{\sin \phi}(x)$ (b) and $A_{U L}^{\sin 2 \phi}(x)(\mathbf{c})$ from a longitudinally polarized proton target for the kinematics of the COMPASS experiment.
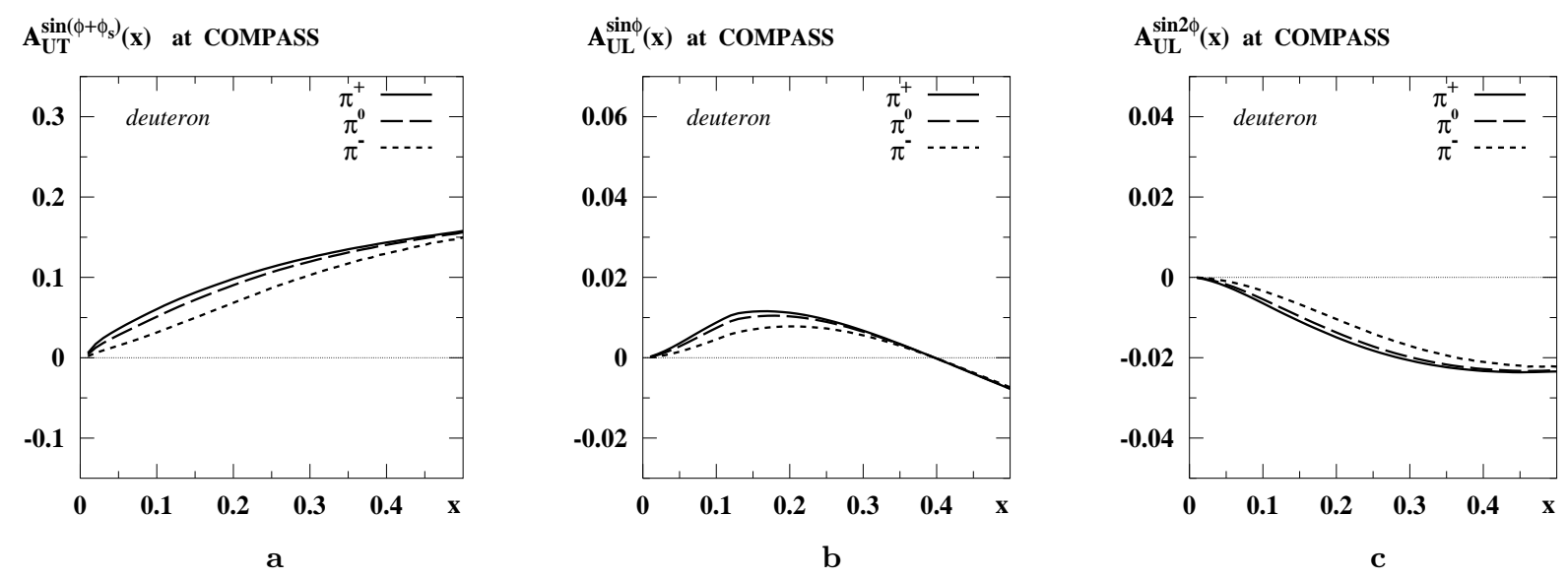

FIG. 4: The same as Fig. 3 but for the deuteron target.

Longitudinal target spin asymmetries. About $80 \%$ of the beam time the target polarization in the COMPASS experiment will be longitudinal [28]. This will allow to measure the longitudinal target spin asymmetries $A_{U L}^{\sin \phi}$ and $A_{U L}^{\sin 2 \phi}$. (In the case of longitudinal target polarization the azimuthal angle of the target polarization vector $\phi_{S}=0$ or $\pi$, cf. Fig. 1.) The estimates for these asymmetries in our approach are shown in Figs. 3b and 3c. Clearly, the longitudinal target spin asymmetries are much smaller than the transverse target spin asymmetry $A_{U T}^{\sin \left(\phi+\phi_{S}\right)}$, however, the larger statistics could help to resolve them.

The $A_{U L}^{\sin 2 \phi}(x)$ asymmetry is of particular interest - since it is one of the "independent observables" which could provide further insights, cf. Section I. This asymmetry was found consistent with zero within error bars at HERMES [1-4]. In our approach at HERMES energies $A_{U L}^{\sin 2 \phi}=\mathcal{O}(1 \%)[22,23]$ - in agreement with the experiment. In the kinematics of the COMPASS experiment we find $A_{U L}^{\sin 2 \phi}(x)=\mathcal{O}(3 \%)$, i.e. of the order of magnitude of the $A_{U L}^{\sin \phi}(x)$ asymmetry observed at HERMES.

Deuteron target. The single target spin asymmetries $A_{U L}^{\sin \left(\phi+\phi_{S}\right)}, A_{U L}^{\sin 2 \phi}$ and $A_{U L}^{\sin \phi}$ for the deuteron target at COMPASS energies are shown respectively in Figs. 4a, 4b and 4c. The deuteron asymmetries for $\pi^{+}, \pi^{0}$ and $\pi^{-}$are all of comparable order of magnitude and about half the magnitude of the $\pi^{+}$proton asymmetries. 


\section{SIVERS EFFECT AZIMUTHAL ASYMMETRIES}

Actually, our approach would imply the vanishing of $A_{U T}^{\sin \left(\phi-\phi_{S}\right)}(x)$ asymmetry, which is due to the Sivers effect [16] and will be measured at HERMES and COMPASS simultaneously with $A_{U T}^{\sin \left(\phi+\phi_{S}\right)}(x)$. However, this cannot be taken literally as a prediction for the following reason. The chiral quark-soliton model was derived from the instanton vacuum model and can be considered as the leading order in terms of the so-called instanton packing fraction $\frac{\rho}{R} \sim \frac{1}{3}(\rho$ and $R$ are respectively the average size and separation of instantons in Euclidean space time). In this order the T-odd distribution functions vanish. In higher orders the Sivers function can be well non-zero and all one can conclude at this stage is that the Sivers function is suppressed with respect to the T-even ${ }^{8}$ twist-2 distribution functions $f_{1}^{a}(x), g_{1}^{a}(x)$ and $h_{1}^{a}(x) .{ }^{9}$ However, considering that $H_{1}^{\perp}(z)$ is much smaller than $D_{1}(z)$, cf. Eq. (13), it is questionable whether such a suppression could be sufficient such that in physical cross sections the Collins effect $\propto h_{1}^{a}(x) H_{1}^{\perp}(z)$ is dominant over the Sivers effect $\propto f_{1 T}^{\perp}(x) D_{1}(z)$. In Ref. [24] it was estimated that for the particular case of $A_{U L}^{\sin \phi}$ asymmetries in the HERMES kinematics this still could be true: Using the Sivers function of Ref. [14] fitted to explain the E704 data [51] on single spin asymmetries in $p p^{\uparrow} \rightarrow \pi X$ solely in terms of the Sivers effect, it was shown that the Sivers effect could give rise to $A_{U T}^{\sin \left(\phi-\phi_{S}\right)}=\mathcal{O}(10 \%)$ while its contribution to $A_{U L}^{\sin \phi}$ is negligible with respect to the Collins effect. Of course, the E704 data need not to be due to the Sivers effect alone, and the Sivers effect in $p p^{\uparrow} \rightarrow \pi X$ need not to be simply related to the Sivers effect in SIDIS. ${ }^{10}$ Therefore the observation of Ref. [24] has to be considered with care. Interestingly, in the quark-diquark model one finds a comparably large $A_{U T}^{\sin \left(\phi-\phi_{S}\right)}=\mathcal{O}(10 \%)[50]$.

To summarize, though in our approach the Sivers functions vanishes, there need not be a contradiction if Sivers effect asymmetry $A_{U T}^{\sin \left(\phi-\phi_{S}\right)}$ would be observed to be sizable. The measurements of $A_{U T}^{\sin \left(\phi \pm \phi_{S}\right)}$ at HERMES and COMPASS (and $A_{U L}^{\sin 2 \phi}$ at COMPASS) will clarify the situation.

\section{CONCLUSIONS}

Recently HERMES observed noticeable azimuthal single spin asymmetries $A_{U L}^{\sin \phi}$ in SIDIS off a longitudinally polarized target [1-4]. These asymmetries could arise from both the Collins and the Sivers effect and are therefore difficult to interpret. Important further insights can be gained from the study of azimuthal asymmetries in SIDIS off a transversely polarized target because the angular distribution of the produced pions allows to cleanly distinguish between the Collins and Sivers effect $[15,16]$.

In this note we have presented estimates of the azimuthal single spin asymmetries due to the Collins effect, $A_{U T}^{\sin \left(\phi+\phi_{S}\right)}$, both for the HERMES and COMPASS experiments. These calculations are based on two ingredients. One ingredient, which is responsible for the $x$-shape of the predicted asymmetries, is the chirally odd transversity distribution function $h_{1}^{a}(x)$ provided by the chiral quark-soliton model ( $\left.\chi \mathrm{QSM}\right)$ [30]. The sign and the overall normalization of the predicted $A_{U T}$ asymmetries are fixed by the second ingredient, namely by properties of the Collins fragmentation function $H_{1}^{\perp}$ resulting from our analysis [22] of the $A_{U L}^{\sin \phi}$ asymmetries observed in the HERMES experiment. On the basis of this approach we estimate the $A_{U T}^{\sin \left(\phi+\phi_{S}\right)}$ to be about $20 \%$ for $\pi^{+}$and $\pi^{0}$ from a proton target and roughly $10 \%$ for all pions from a deuterium target.

Choosing another weight, namely $\sin \left(\phi-\phi_{S}\right)$, it is possible to project out another azimuthal asymmetry which is due to the Sivers effect only [16]. If taken literally, our approach would predict a vanishing Sivers effect asymmetry $A_{U T}^{\sin \left(\phi-\phi_{S}\right)}$ because in the $\chi \mathrm{QSM}$ the Sivers distribution function vanishes. This shortcoming is met basically in all chiral effective models [39] and reflects the limitations of such models to describe T-odd distribution functions. In the $\chi$ QSM, which is based on an expansion in terms of the packing fraction of the instantons in the vacuum, T-odd distribution functions are subleading quantities in contrast T-even distribution functions. However, a Sivers function as large as obtained in the quark-diquark models with gluon exchange $[12,49,50]$ yielding $A_{U T}^{\sin \left(\phi-\phi_{S}\right)}=\mathcal{O}(10 \%)[50]$ would not be in contradiction with our approach [24].

\footnotetext{
8 The suppression of T-odd with respect to T-even distributions is natural. E.g. in the quark-diquark models with gluon exchange $[12,49,50]$ - where the Sivers function was "rediscovered", cf. [11] - T-even distributions appear at the tree-level while T-odd ones appear only at one-loop level. Thus, whatever (small) parameter justifies the perturbative calculation of distribution functions in the quark-diquark model, it generically suppresses T-odd distributions with respect to T-even ones.

${ }^{9}$ In the case of the pure twist-3 $\widetilde{h}_{L}^{a}(x)$ [38] (or $\widetilde{g}_{T}^{a}(x)$ [36]) it was shown on the basis of [52] that the suppression in the instanton medium with respect to twist-2 distributions is very strong.

10 Cf. the corresponding discussions of the Sivers effect in SIDIS and the Drell-Yan process, where the Sivers functions differ by an overall sign $[11,13]$.
} 
Noteworthy, the longitudinal target polarization program of the COMPASS experiment may also well contribute to the understanding of single spin asymmetries in SIDIS. Our approach predicts the $A_{U L}^{\sin 2 \phi}$ asymmetry, which was found consistent with zero within (relatively large) error bars at HERMES, is of $\mathcal{O}(3 \%)$ in the COMPASS kinematics and can probably be resolved. This asymmetry is due to the Collins effect only and its measurement would provide valuable independent information. The $A_{U L}^{\sin \phi}$ asymmetry is about $(1-2) \%$ and more difficult to measure for COMPASS.

A measurement of the $A_{U T}^{\sin \left(\phi+\phi_{S}\right)}$ asymmetry at HERMES and COMPASS and the $A_{U L}^{\sin 2 \phi}$ at COMPASS of comparable magnitude as we estimated here would support the observation [24] that the Sivers effect could play a sub-dominant role in the $A_{U L}^{\sin \phi}$ asymmetries measured by HERMES [1-4] and a posteriori justify the attempts [17-23] to interpret these data in terms of the Collins effect only. In contrast, deviations from our predictions could provide valuable hints how those attempts should be modified. We will - in any case - soon learn a lot from the HERMES and COMPASS experiments.

Acknowledgement. We are grateful to H. Avakian, M. Beckmann, I. Ludwig, R. Seidl for fruitful discussions. A. E. is partially supported by INTAS grant 00/587 and RFBR grant 03-02-16816 and DFG-RFBR 03-02-04022. This work has partly been performed under the contract HPRN-CT-2000-00130 of the European Commission. The work is partially supported by BMBF and DFG of Germany and by the COSY-Juelich project.

\section{APPENDIX A: EXPRESSIONS FOR LONGITUDINAL TARGET POLARIZATION ASYMMETRIES}

For the convenience of the reader we summarize the expressions for $A_{U L}^{\sin 2 \phi}$ and $A_{U L}^{\sin \phi}$ which were derived in $[19,22,23]$ on the basis of the results from Ref. [15]:

$$
\begin{aligned}
A_{U L}^{\sin \phi}(x) & =a_{\text {Gauss }}\left(P_{L}(x) \frac{\sum_{a} e_{a}^{2} x^{2} h_{L}^{a}(x)\left\langle H_{1}^{\perp a}\right\rangle}{\sum_{b} e_{b}^{2} x f_{1}^{b}(x)\left\langle D_{1}^{b}\right\rangle}+P_{1}(x) \frac{\sum_{a} e_{a}^{2} x h_{1}^{a}(x)\left\langle H_{1}^{\perp a}\right\rangle}{\sum_{b} e_{b}^{2} x f_{1}^{b}(x)\left\langle D_{1}^{b}\right\rangle}\right) \\
A_{U L}^{\sin 2 \phi}(x) & =4\langle z\rangle^{2} a_{\text {Gauss }}^{2} \frac{2 M_{N}}{\left\langle P_{\perp h}\right\rangle} P_{2}(x) \frac{\sum_{a} e_{a}^{2} 2 x^{3} \int_{x}^{1} \mathrm{~d} y h_{1}^{a}(y) / y^{2}\left\langle H_{1}^{\perp a}\right\rangle}{\sum_{b} e_{b}^{2} x f_{1}^{b}(x)\left\langle D_{1}^{b}\right\rangle}
\end{aligned}
$$

where $a_{\text {Gauss }}$ is defined as in Eq. (9) and the $P_{i}(i=L, 1,2)$ are given by

$$
\begin{aligned}
P_{L}(x) & =\frac{2 \int \mathrm{d} y 2(2-y) \sqrt{1-y} \cos \theta_{\gamma} M_{N} / Q^{5}}{\int \mathrm{d} y\left(1-y+y^{2} / 2\right) / Q^{4}}, \\
P_{1}(x) & =-\frac{2 \int \mathrm{d} y(1-y) \sin \theta_{\gamma} / Q^{4}}{\int \mathrm{d} y\left(1-y+y^{2} / 2\right) / Q^{4}}, \\
P_{2}(x) & =\frac{2 \int \mathrm{d} y(1-y) \cos \theta_{\gamma} / Q^{4}}{\int \mathrm{d} y\left(1-y+y^{2} / 2\right) / Q^{4}} .
\end{aligned}
$$

[1] H. Avakian [HERMES Collaboration], Nucl. Phys. Proc. Suppl. 79 (1999) 523.

[2] A. Airapetian et al. [HERMES Collaboration], Phys. Rev. Lett. 84 (2000) 4047.

[3] A. Airapetian et al. [HERMES Collaboration], Phys. Rev. D 64 (2001) 097101.

[4] A. Airapetian et al. [HERMES Collaboration], Phys. Lett. B 562 (2003) 182.

[5] H. Avakian et al. [CLAS Collaboration], arXiv:hep-ex/0301005.

[6] A. Bravar [Spin Muon Collaboration], Nucl. Phys. Proc. Suppl. 79 (1999) 520.

[7] J. C. Collins, Nucl. Phys. B 396 (1993) 161.

X. Artru and J. C. Collins, Z. Phys. C 69 (1996) 277.

[8] D. W. Sivers, Phys. Rev. D 41 (1990) 83; Phys. Rev. D 43 (1991) 261.

[9] J. Ralston and D. E. Soper, Nucl.Phys B152 (1979) 109.

J. L. Cortes, B. Pire and J. P. Ralston, Z. Phys.C55 (1992) 409.

R. L. Jaffe and X. Ji, Phys. Rev. Lett. 67 (1991) 552; Nucl. Phys B375 (1992) 527.

[10] A. V. Efremov, L. Mankiewicz and N. Törnqvist, Phys. Lett. B284 (1992) 394.

[11] J. C. Collins, Phys. Lett. B 536 (2002) 43.

[12] S. J. Brodsky, D. S. Hwang and I. Schmidt, Phys. Lett. B 530 (2002) 99.

[13] A. V. Belitsky, X. Ji and F. Yuan, Nucl. Phys. B 656 (2003) 165. X. D. Ji and F. Yuan, Phys. Lett. B 543 (2002) 66. 
[14] M. Anselmino, M. Boglione and F. Murgia, Phys. Lett. B 362 (1995) 164.

M. Anselmino and F. Murgia, Phys. Lett. B 442 (1998) 470.

[15] P. J. Mulders and R. D. Tangerman, Nucl. Phys. B 461 (1996) 197 [Erratum-ibid. B 484 (1997) 538].

[16] D. Boer and P. J. Mulders, Phys. Rev. D 57 (1998) 5780.

[17] E. De Sanctis, W. D. Nowak and K. A. Oganesian, Phys. Lett. B 483 (2000) 69.

K. A. Oganessian, N. Bianchi, E. De Sanctis and W. D. Nowak, Nucl. Phys. A 689 (2001) 784.

[18] M. Anselmino and F. Murgia, Phys. Lett. B 483 (2000) 74.

[19] A. V. Efremov, K. Goeke, M. V. Polyakov and D. Urbano, Phys. Lett. B 478 (2000) 94.

[20] B. Q. Ma, I. Schmidt and J. J. Yang, Phys. Rev. D 66 (2002) 094001; Phys. Rev. D 65 (2002) 034010; Phys. Rev. D 63 (2001) 037501.

[21] V. A. Korotkov, W. D. Nowak and K. A. Oganesian, Eur. Phys. J. C 18 (2001) 639.

[22] A. V. Efremov, K. Goeke and P. Schweitzer, Phys. Lett. B 522 (2001) 37 [Erratum-ibid. B 544 (2002) 389].

[23] A. V. Efremov, K. Goeke and P. Schweitzer, Eur. Phys. J. C 24 (2002) 407; Nucl. Phys. A 711 (2002) 84; Acta Phys. Polon. B 33 (2002) 3755.

[24] A. V. Efremov, K. Goeke and P. Schweitzer, Phys. Lett. B 568 (2003) 63.

[25] A. V. Efremov, K. Goeke and P. Schweitzer, Phys. Rev. D 67 (2003) 114014. For a recent overview on $e^{a}(x)$ see: A. V. Efremov and P. Schweitzer, JHEP 0308 (2003) 006.

[26] H. Avakian, talk at "8th Conference On The Intersections Of Particle And Nuclear Physics (CIPANP 2003)", 19-24 May 2003, New York, New York.

[27] N. C. Makins and M. Düren [HERMES Collaboration], Acta Phys. Polon. B 33 (2002) 3737. N. C. Makins [HERMES Collaboration], Nucl. Phys. A 711 (2002) 41.

[28] J. M. Le Goff [COMPASS Collaboration], Nucl. Phys. A 711 (2002) 56. M. Lamanna [COMPASS Collaboration], Nucl. Phys. A 711 (2002) 50.

[29] A. Bacchetta, R. Kundu, A. Metz and P. J. Mulders, Phys. Rev. D 65 (2002) 094021.

[30] P. V. Pobylitsa and M. V. Polyakov, Phys. Lett. B 389 (1996) 350.

P. Schweitzer et al., Phys. Rev. D 64 (2001) 034013.

[31] J. C. Collins, Acta Phys. Polon. B 34 (2003) 3103.

[32] For reviews see: D. I. Diakonov, Prog. Part. Nucl. Phys. 51 (2003) 173. D. I. Diakonov and V. Y. Petrov, Contribution to the Festschrift in honor of B. L. Ioffe, ed. by M. Shifman [arXiv:hep-ph/0009006].

[33] C. V. Christov et al., Prog. Part. Nucl. Phys. 37 (1996) 91.

[34] D. I. Diakonov, V. Yu. Petrov, P. V. Pobylitsa, M. V. Polyakov and C. Weiss, Nucl. Phys. B 480 (1996) 341.

[35] D. I. Diakonov et al., Phys. Rev. D 56 (1997) 4069. P. V. Pobylitsa et al., Phys. Rev. D 59 (1999) 034024. C. Weiss and K. Goeke, preprint RUB-TPII-12-97 [arXiv:hep-ph/9712447]. K. Goeke et al., Acta Phys. Polon. B 32 (2001) 1201.

[36] J. Balla, M. V. Polyakov and C. Weiss, Nucl. Phys. B 510 (1998) 327.

[37] A. Y. Umnikov, H. X. He and F. C. Khanna, Phys. Lett. B 398 (1997) 6.

[38] B. Dressler and M. V. Polyakov, Phys. Rev. D 61 (2000) 097501.

[39] P. V. Pobylitsa, arXiv:hep-ph/0212027.

[40] A. Schäfer and O. V. Teryaev, Phys. Rev. D 61 (2000) 077903.

[41] A. V. Efremov, O. G. Smirnova and L. G. Tkachev, Nucl. Phys. Proc. Suppl. 74, 49 and 79, 554 (1999).

[42] A. V. Efremov, Y. I. Ivanshin, O. G. Smirnova, L. G. Tkachev and R. Y. Zulkarneev, Czech. J. Phys. 49 (1999) S75 [arXiv:hep-ph/9901216].

[43] D. Boer, Nucl. Phys. B 603 (2001) 195.

[44] D. Boer, P. J. Mulders and F. Pijlman, Nucl. Phys. B 667 (2003) 201.

[45] M. Glück, E. Reya and A. Vogt, Z. Phys. C 67 (1995) 433.

[46] A. D. Martin, R. G. Roberts, W. J. Stirling and R. S. Thorne, Eur. Phys. J. C 4 (1998) 463.

[47] P. Abreu et al. [DELPHI Collaboration], Z. Phys. C 73 (1996) 11.

[48] J. Soffer, Phys. Rev. Lett. 74 (1995) 1292. G. R. Goldstein, R. L. Jaffe and X. D. Ji, Phys. Rev. D 52 (1995) 5006.

[49] S. J. Brodsky, D. S. Hwang and I. Schmidt, Phys. Lett. B 553 (2003) 223; Nucl. Phys. B 642 (2002) 344. D. Boer, S. J. Brodsky and D. S. Hwang, Phys. Rev. D 67 (2003) 054003.

[50] L. P. Gamberg, G. R. Goldstein and K. A. Oganessyan, Phys. Rev. D 67 (2003) 071504.

[51] D. L. Adams et al. [FNAL-E704 Collaboration], Phys. Lett. B 264 (1991) 462; Phys. Lett. B 276 (1992) 531; Z. Phys. C 56 (1992) 181.

[52] D. Diakonov, M. V. Polyakov and C. Weiss, Nucl. Phys. B 461 (1996) 539. 